Monatsschrift für Geburtshülfe u. Gynäkologie 1907;26:876-883

\title{
Literatur-Verzeichnis
}

\section{Prof. $\mathrm{Ph} . \ll / \mathrm{Mr} / 8 \mathrm{r}-$ Greifswald.}

I. Geburtshülfe.

Allgemeines.

Bacon, Gh. S., The relations of the teaching of clinical obstetrics to the problem of med. charities. Illinois med. Journ. September.

Literatur - Verzeichnis.

877

Keller, A., Zur Ammenwahl und Ammenbehandlung. Berl. klin. Wochen-

schrift. No. 40. 7. Oktober. S. 1296. Rissmann, P., Die Vereinigung zur Förderung des deutsehen Hebammen-

wesens. Gyn. Rundschau. No. 20. Walther, H., Ein Beitrag zur sozialen Lage der Hebammen. Gyn.

Rundschau. No. 19.

Physiologie und Pathologie der Schwangerschaft, der Geburt und des Wochenbettes.

Acconcí, G., I fermenti proteolitici della placenta: loro valore biologico. La Rassegna d'Ostetr. e ginec. Napoli. Oktober.

Aïbeck, V., Bakteriurie und Pyurie bei Schwangeren und Gebärenden. Zeitschr. f. Geb. u. Gyn. Bd. 60 .

Baffoni-Luciani, F., Placenta angolare. La Ginecologia. 15. August.

Baisch, K., Ursache, Verhütung und Behandlung des Kindbettfiebers. Beihefte zur med. Klinik. III. Jahrg. H. 10.

Bircher, E., Ovarialsarkom während der Gravidität. Centralbl. f. Gyn. No. 44.

Blau, A., Zur Entstehung der subchorialen Cysten der Placenta. Zeit-schrift f. Geb. u. Gyn. Bd. 60 .

Bouchacourt, Radiopelvimétrie d'un bassin oblique ovalaire de Naegelé. Bulletin d. 1. Société d'obstétrique de Paris. No. 7 u. 8. 20. Juni u. 4. Juli.

ter Braak, I. 0., und Mijnlíeff, A., Ein Fall von Eklampsie infolg $\theta$ erhöhter intrarenaler Spannung. Centralbl. f. Gyn. No. 42.

Gova, E., Sulle cosi dette cellule del Langhans periferiche $\theta$ i loro rapporti coi vasi. La Ginecologia. 15. August.

Davis, E. P., The delivery of debilitated women, with especial reference to the interest of the child. Journ. of Amer. med. Ass. 19. Oktober.

Eshner, A. A., The relations between diabetes and pregnancy: with the report of a case of diabetes, in which the glycosuria disappeared with the inception of pregnancy and reappeared after delivery. Amer. Journ. of med. Sciences. September.

Ferraro, Cisti ovariche e gravidanza. La Rassegna d'Ostetr. e Gynecologia. No. 9. September. Frank, Robert T., Results obtained by the injection of placenta into animals of the same and of different species. (From the Rockefeller-Institute for med. Research, New York.) The Journ. of experimental Medicin. Vol. IX. No. 3. Mai. 
Fruhinsholz, M. A., Des lesions suppuratives développées dans les membranes de Гoeuf. Ann. de Gyn. Oktober.

Gache, S., Trois cas d'inversion puerpérale. Ann. de Gyn. Oktober.

Guicciardi, G., Tuberculosi e gravidanza. La Ginecologia. 31. August.

Halban, I., Zur Frage der Graviditäts-Hypertrichose. Wien. klin. Wochen-schrift. No. 45.

Hiltor, David C, Appendicitis complicating the puerperium including a review of the litterature of reported cases. Surgery, Gynecology and Obstetrics. S. 441. Oktober.

Hofbauer, J., Die menschliche Placenta als Assimilationsorgan. Samml. klin. Vortr. N.-F. No.

454 (Serie XVI, H. 4). Gynäkologie. No. 166.

878

Literatur -Verzeichnis.

Jung, Ph., Neue Bestrebungen in der Behandlung der puerperalen In-

fektion. Med. Klinik. No. 42. Kaessmann, F, Ein Beitrag zur Adrenalinbehandlung der

Osteomalakie

nach Bossi. Centralbl. f. Gyn. No. 43. McKey, St., The effect of blood transfusion on a patient with puerperal

eclampsia. Amer. Journ. of Obst. Oktober. Merletti, Nuovi elementi por lo studio della funziona lità cardiaca nello

stato puerperale. La Rassegna d'Obstetr. e Ginecol. Napoli. Oktober. Michel de Kewily, Une lesion très fréquente du fois chez les éclamptiques:

Surcharge graisseuse des cellules étoilées. Bull. soc. d'Obstétrique.

Paris. No. 7 u. 8. 20. Juni u. 4. Juli. Opocher, Ií glicogene contenuto nel fegato materno in gravidanza ed

in puerperio, nella placenta e nel fegato fetale. La Rassegna d'Ostetr.

e di Ginecol. No. 9. September. Rebaudi, St., Blood platelets during pregnancy, labour, the

puerperium

and menstruation, and in the new born. Amer. Journ, of Obst.

Oktober. Reed, Char. B., Gangrene and Eclampsia. Surgery, Gynecology and

Obstetrics. Vol. 5. No. 3. S. 326. September. Rissmann, P., Adstringentien und prophylaktische

Scheidenspülungen.

Gyn. Rundschau. No. 19. Strempel, Beitrag zur Behandlung der Eklampsie im Wochenbett nach Bumm. Centralbl. f. Gyn. No. 43. Schmidt, H., Über die Verwendung der

flüssigenSomatosebeiWöchnerinnen.

Munch, med. Wochenschr. No. 42. Winston, J. W., Management of the third stage of labour.

Med. Record.

12. Oktober.

Abortus. - Geburtshülfliche Operationen. -

Operationen im Wochenbett.

Baguel, Andre, Fibrome praevia. Operation Césarienne à terme suivie

d'hysterectomie abdominale subtotale. Bull. Soc. d'Obstétrique.

Paris. No. 7 u. 8. 20. Juni und 4. Juli. Bokelmann, W. , Zur Frage der künstlichen Unterbrechung der Schwanger-

schaft bei inneren und Geisteskrankheiten. S Samml. zwangl. Abh.

M. Graefe. Halle, C. Marhold. Bosni, L. M,, Die künstliche Geburt mittelst der schnellen

mechanischen

Erweiterung des Uterushalses und die mechanischen Dilatatoren. 
Gyn. Rundschau. No. 20. Good, Frederick J., A new obstetric forceps. Surgery, Gynecology, Obstetrics. Vol. V. No. 3. S. 342. September. Gushee, E. S., Some observations on craniotomy. Bulletin lying' in

Hosp. city of New York. Vol. IV. Juni. Knauer, E., Bericht über vier an der Grazer Frauenklinik aus-

geführte Hebosteotomien, nebst einigen Bemerkungen zur Technik und lndikation dieser Operation. Mitteilungen d. Ver. d. Ärzte in Steiermark. No. 10 u. 11. Lepage, De la valeur du traitement des vomissements graves de la grossesse par la dilatation du col de Гutérus. Comptes rendus de la Société d'Obstétrique de Gynécologie et de Pädiatrie. Tome IX. Paris. S. 228. Oktober. Literatur - Verzeichnis. 879 Mann, R., Zur Hernienbildung nach Hebosteotomie. Centralbl. f. Gyn.

No. 44. di Nardo, Parto cesarea col taglia trasversale sul fondo delPutero nel rachitismo e nell osteomalacia. La Rassegna d'Ostetricia e Gynecologia. No. 9. September. Raineri, G., Experimenteller Beitrag zum Studium der dauernden Beckenerweiterung nach Hebosteotomie. Centralbl. f. Gyn. No. 45. Derselbe, Contributo sperimentale allo studio dellallargamento permanente del bacino dopo pubiotomia. La Rassegna d'Ostetricia e Génecologia.

Napoli. Oktober. Reynolds, E., Constitutional ill-equipment of the patient as a factor in determining the performance of the primary cesarean section.

The Journ. of Amer. Ass. 19. Oktober. Routh, A., A case of cesarean hysterectomy for traumatic atresia of

the vagina, the patient having previously undergone a successful operation for a vesico-vaginal fistula due to the same injury.

Journ. of Obst. and Gyn. of Brit. Emp. November. Sinclair, W., Caesarean section successfully performed for the fourth

time on the same woman, with remarks on the production of utero-

parietal adhesions. Journ. of Obst. and Gyn. of Brit. Emp.

November. Sitzenfrey, A., Parovariotomia vaginalis sub partu. Prager med. Wochenschrift. No. 44.

Fötus. - Neugeborenes. Cuzzi, A., Sulla eziologia e sulla patogenesi dello svilleppo extracoriale del feto. La Ginecologia. Anno IV. Firenze. Falk, E., Zum Umformungsprozess der Wirbelsäule während der fötalen

Entwicklung. Berl. klin. Wochenschr. No. 45. 11. November. Gilles, Dystocie foetale par dégénérescence kystique des reins. Bull. soc.

Obstétrique, Paris. No. 8. 4. Juli. Grosse, Un cas d'accouchement triple. Comptes rendus de la Société d'Obstétrique, de gynécologie et de Paediatric. Tome IX. Paris.

S. 241. Oktober. Guéniot, Paul, Quelques notes bactériologiques sur 11 cas de coryza épidémique des nouveau-nés. Bullet. Soc. d'Obstétrique. Paris.

No. 8. 4. Juli. v. Herff, 0., Kami man die Zahl der Erkrankungen an Ophthalmoblennorrhoea gonorrhoica verringern? Gyn. Rundschau. No. 19. Levi, J., Di un aspiratore delle mucosità nell asfissie dei neonati. 
La Rassegna d'Ostetr. e Ginec. Napoli. Oktober. Markoe, J. W., An unusual malformation of the cranial bones in a newborn infant. Bull, of the Lying-in Hospital of the City of New-York. Vol. IV. Juni. Maygrier und Schwab, Histoire d'un petit premature, du poids $840 \mathrm{~g}$.

Bull. Société d'Obstétrique. Paris. No. 7 und 8. 20. Juni und

4. Juli. Moro, E., Experimentelle Beiträge zur Frage der künstlichen Säuglings-

ernährung. Munch, med. Wochenschr. No. 45. Pfaundler, M., und Moro, E., Zur Physiologic und Pathologie der Säuglings-

ernährứg. Munch, med. Wochenschr. No. 44.

880

Literatur - Verzeichnis.

Pohlman, A. G., The fetal circulation through the heart. A review of the more important theories, together with a preliminary report on personal findings, Bull, of the Johns Hopkins Hosp.

Baltimore. Oktober.

Printing, F., Die örtlichen Verschiedenheiten der Zwillingshäufigkeit und deren Ursachen.

Zeitschr. f. Geb. und Gyn. LX. Bd.

Ektopische Schwangerschaft.

Folk, E., Ein Fall von Eversion der Tube bei tubarem Abort. Centralbl. f. Gyn. No. 45.

Guerdikoff, M. N., Manoeuvres abortives au cours de la grossesse extra-utérine. Ann. de Gyn.

Oktober.

Kelley, J. Th., Ectopic pregnancy. Amer. Journ. of Obst. Oktober.

Maygrier, Grossesse interstitielle. Rupture à 2 mois $1 / 2$ environ, hémor-rhagie et péritonite.

Laparotomie et hysterectomie. Mort (presentation de la piece). Bull. Soc. d'Obst. Paris. No. 8. 4. Juli.

Mursell, H. T., A case of retroperitoneal haematocele producing acute intestinal obstruction. Brit. med. Journ. 12. Oktober.

Taylor, E. C, Ectopic pregnancy. Amer. Journ. of Surg. September. S. 278.

Wagner, A., Die Extrauterin-Schwangerschaft. Klinische Studie axif Grund von 32

selbstbeobachteten Fallen. Stuttgart. F. Enke.

II. Gynäkologie.

Allgemeines.

Alsberg, P., Hyperalgesien der Haut in ihrer Bedeutung fur die Gynäkologie. (Frauenklinik L.

und Th. Landau.) Deutsche med. Wochenschr. No. 45. 7. November.

Chase, W. B., The time factor in surgical operations. Amer. Journ. of Obst. Oktober.

Daels, Franz, Experimenteller Beitrag zur Wirkung des Yohimbin auf den weiblichen

Genitalapparat. (Aus der experimentell-biologischen Abteilumg des königl. pathol. Institute der Universität Berlin.) Berl. klin. Wochenschr. 21. Okt. No. 42. S. 1332.

Fitch, W. E., Correction of faulty fashions in dress an important factor in the treatment of diseases of women. Med. Record. 19. Oktober.

Lewis, D., Practical venereal prophylaxis. Med. Record. 12. Oktober.

Micheli, Nuovo metodo di respirazione artificiale. La Rassegna d'Ostetr. e ginecol. September.

No. 9.

Morley, W. H., Ann Arbor. Post-operative Thrombophlebitis. Surg. Gyn. and Obstetr.

September. Vol. V. No. 3. S. 299.

Pankow, Das Alttuberkulin Koch als Diagnosticum in der Gynäkologie. Centralbl. f. Gyn. No. 42. 
Ross, Mc. Pherson, Some improvements in operating room construction at the lying-in hospital and an ingenious method of securing additional floor in space. Bull, of Lying-in Hosp. City of New-York. Juni. Vol. IV.

Stratz, C. H,, Die körperliche Pflege der Frau. Physiologische und ästhetische Diätetik für das weibliche Geschlecht. Allgemeine

Literatur-VerzOichnis. 881

Körperpflege, Kindheit, Reife, Heirat, Ehe, Schwangerschaft, Geburt, Wochenbett, Wechseljahre. F. Enke. Stuttgart. Weinberg, S., Ueber den Einfluss der Geschlechtsfunktionen auf die weibliche Kriminalität. Juristisch-psychiatrische Grenzfragen. Zwang-lose Abhandl. Finger, Hoche, Bresler. VI. Bd. Heft 1. Halle. C. Marhold.

Uterus und Operationen an demselben.

Barberio, M., Ueber einen höchst seltenen Fall von Calcifikation de Uteruswand, besonders in der Umgebung der Uterushöhle. Zeitschr.

f. Geb. und Gyn. LX. Bd. v. Bardeleben, H., Die klinischen Formen und die Behandlung der Endo-

metritis. Die Therapie der Gegenwart. 11. Heft. Oktober. Freund, H. W., Zur Behandlung der Dysmenorrhoe von den Brüsten

aus. Munch, med. Wochenschr. No. 43. Fuchs, A., Polymorphzellige Tumoren der Cervix uteri. Zeitschr. für

Geb. und Gyn. LX. Bd. Mansfeld, 0. P., Zur Diagnose der Malignität am Uterus. Zeitschr. für Geb. und Gyn. LX. Bd. Schenk, F., und Sitzenfrey, Gleichzeitiges Karzinom des Magens, der Ovarien und des Uterus, mit besonderer Berücksichtigung ihrer operativen Behandlung und der histologischen Befunde. Zeitschr.

f. Geb. und Gyn. LX. Bd. Schmauch, Geo., The histologic and clinical significance of malignant chorioepithelioma. Surgery, Gynec. and Obst. Vol. V. No. 3.

S. 259. September. Schütze, A., Eine seltene Beobachtung der Kombination von Karzinom, diffusem Adenom und Tuberkulose, nebst Bildung von Psammomkörpern im Uterus seiner 40jährigen Nullipara. Zeitschr. f. Geb. und Gyn. LX. Bd. Stone, S., Chorionepithelioma. Amer. Jo urn. of Obst. Oktober. Strassmann, P., Die operative Vereinigung eines doppelten Uterus.

Centralbl. f. Gyn. No. 43. Waiss, George C, Hysterectomy for hemorrhage. Report of a case of vaginal hysterectomy for uncontrollable uterine hemorrhage due to pregnancy changes at the placental part of the uterus. Surgery, Gynec. and Obst. Chicago. S. 438. Oktober.

Ovarien. - Tuben. - Ligamente.

Gasalis, G. A., A case, in which ligature of the fallopian tubes failed to effect sterilization. Journ. of Obst. and Gyn. of Brit. Emp.

November. Crons, J. J., Polycystic lutein degeneration of the ovaries. Amer.

Journ. of Obst. Oktober. Mann, E. C, Ruptured pus tubes. Amer. Journ. of Obst. Oktober. Oliver, J., A study of suppurations of the ovary with illustrative cases.

Journ. of Obst. and Gyn. of Brit. Emp. November. Taylor, F. E., Typhoid infection of ovarian cysts. Journ. of Obst. and

Gyn. of Brit. Emp. November.

882

Literatur-Verzeichnis.

Vulva. - Scheide. - Damm. - Beckenbindegewebe. 
Cohn, Ein neues Pessar gegen Prolaps. Berlin, klin. Wochenschr. 1907.

No. 44. 4. November. Gross, E., Multiple gutartige Geschwülste der Vulva. Zeitschr. f. Geb. und Gyn. LX. Bd. Kuntzsch, Kohabitationshindernis infolge von Hymenalcyste und Vaginismus. Konzeption trotz fehlender Defloration. Centralbl. f. Gyn.

No. 45.

Bauchhöhle. - Bauchschnitt. - Scheidenbauchschnitt.

Beer, E., A note on the sensibility of the human visceral peritoneum.

Med. Record. 5. Oktober. Bovée, J. W., The scope of treatment of acute pelvic infections in women by general physician. Amer. Journ. of Obst. Oktober. Brewer, G. E., The etiology of certain cases of left-sided intra-

abdominal suppuration - acute diverticulitis. Amer. Journ. of med.

Sciences. Oktober. Galdesi, Del chemaggio peritoneale post-laparotomico. La Rassegna

d'Ostetricia e Gynecol. No. 9. September. Gumston, Gh. G., Ptosis of the abdominal viscera surgically considered.

Med. Record. 19. Oktober. Funck-Brentano, Production immediate d'une grande quantité de gaz dans la cavité péritonéale et sous le péritoine, dans presque toute

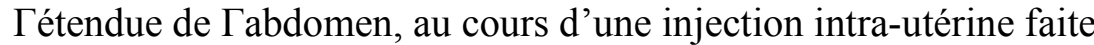

avec de Гeau oxygénée. Comptes-rendus de la Société d'Obstétrique,

de Gynécologie et de Paediatrie. Tome IX. Oktober. Paris. S. 226. Johnstone, R. J., Recurrence

of retroperitoneal lipoma: Operation,

recovery. Brit. med. Journ. 12. Oktober. Kothe, R.; Zur Behandlung der diffusen,

fortschreitenden Peritonitis

mittelst permanenter rektaler Kochsalzinfusionen. Die Therapie

der Gegenwart. H. 10. Oktober. Kreucker, E., Bacterium coli commune als Sepsiserreger in 2

Fallen von

Abdominalerkrankungen. Munch, med. Wochenschr. No. 42. Wathek, William H., Acute

suppurative peritonitis (local, speading,

diffuse and general). Journ. of the Amer. med. Assoc. 8. Juni.

Harnorgane. - Fisteln.

Buckhardt, L., und Polano, 0., Die Untersuchungsmethoden und Er-

krankungen der männlichen und weiblichen Harnorgane, für Ärzte

und Studierende in 17 Vorlesungen. Wiesbaden. J. F. Bergmann. v. Frankl-Hochwart, L., Zur

Differentialdiagnose der juvenilen Blasen-

störungen. Zugleich ein Beitrag der Kenntnis des spinalen Blasen-

tumors. Wiener klin. Rundschau. No. 43. Goldschmidt, H., Betrachtungen über die Endoskopie der Harnröhre.

Die Therapie der Gegenwart. H. 11. November. Hannes, W., Cystoskopische Befunde bei

operativ geheilten Blasen-

Scheidenfisteln. Centralbl. f. Gyn. No. 43. Kallmann, A., und Jaeoby, S., Jahresbericht über die Leistungen und

Fortschritte auf dem Gebiete der Erkrankungen des Urogenital-

apparats. 2. Jahrgang. S. Karger-Berlin.

Literatur-Verzeichnis. 883

Munow, J. C, und Goddard, S. W., Pyelonephrosis of a supernumerary

kidney. Amer. Journ. of med. Sciences. September. Riedel, Über die verschobene, an falsehem Orte durch Verwachsungen 
festgelegte rechte Niere (Schluss aus No. 41). Deutsche med.

Wochenschr. No. 42. 17. Oktober. Sternberg, C, Zur Kasuistik der Nierendefekte und

Missbildungen des

Urogenitalapparates. Wiener klin. Wochenschr. No. 45. Thomson, A., The making of a shelf below the unduly mobile kidney.

Edinburgh med. Journ. Oktober.

Darm. - Hernien. Harris, M. L., The paraffin injection treatment of inguinal hernia.

Journ. of Amer. med. Assoc. 19. Oktober. Mc. Plowright, Ch. T., Malignant disease of the intestine rendering on

inguinal hernia irreducible. Brit. med. Journ. 12. Oktober. Reynier und Tissier, Hernie

embryonnaire (presentation de la pièe).

Bull. soc. Obst. Paris. No. 8. 4. Juli.

Mamma. Miles, A., Resume of some recent papers on the ultimate results of

operations for cancer of the breast. Edinburgh med. Journ.

Oktober. Perking, W. C, Fibroma molluscum and pendent tumor of the breast.

Med. Record. 5. Oktober.

Narkose. - Anti- und Asepsis.

Physiologische Gesellschaft zu Berlin. 7. Juni 1907.

Brat, Über den plötzlichen Tod in der Narkose. Deutsche med. Wochen-

schrift. No. 42. 17. Oktober. Fischer, J., Historisches zur Verwendung der Handschuhe in der Ge-

burtshülfe. Gyn. Rundschau. No. 20. Grasmann, M., Versuche über Händedesinfektion mit besonderer Be-

rücksichtigung der von Heusner empfohlenen Jodbenzinmethode.

Munch, med. Wochenschr. No. 42, 43. Meyer, F., und Rappel, W. G., Über Streptokokken und Antistreptokokken-

serum. Med. Klinik. No. 40. 6. Oktober. Remenär, A., Über Rückenmarks-Anästhesierung.

Wiener klin. Wochen-

schrift. No. 45. Rose, A., Asepsis and non Asepsis. Amer. Journ. of Obst. Oktober. Bchumburg, Neuere Erfahrungen über Händedesinfektion. Zeitschr. f.

ärztl. Fortbildung. No. 19. Thaler, H., Zur Asepsis bei Laparotomien. Wiener klin. Wochenschr. No. 42.

Technisches. - Akiurgie. Jacobsen, J. H., A simple and refective self-retaining abdominal retractor.

Surgery, Gyn. und Obst. Oktober 1907. Chicago. S. 447. Scherbak, A. L., Der Simonsche Spatel als selbsthaltendes Speculum.

Centralbl. f. Gyn. No. 45.

Verantwortlicher Redakteur: A. Martin in Berlin.

Monatsschrift für Geburtshülfe u. Gynäkologie. Bd. XXVI. Heft 6. 58 